\title{
Ionospheric Correction and Ambiguity Resolution in DGPS with Single Frequency
}

\author{
Norsuzila Ya’acob \\ Department of Electrical, Electronics and Systems Engineering \\ Universiti Kebangsaan Malaysia \\ 43600 UKM Bangi, Selangor, Malaysia \\ Tel: 19-333-0864Ｅ-mail: norsuzilayaacob@yahoo.com \\ Mardina Abdullah and Mahamod Ismail \\ Department of Electrical, Electronics and Systems Engineering \\ UniversitiKebangsaan Malaysia \\ 43600 UKM Bangi, Selangor, Malaysia \\ Tel: 60-3-8921-6304 E-mail: mardina@eng.ukm.my; mahamod@eng.ukm.my \\ Kamaruzaman Jusoff (Corresponding author) \\ Department of Forest Production, Faculty of Forestry \\ Universiti Putra Malaysia, 43400, UPM, Serdang, Selangor, Malaysia \\ Tel: 60-3-8946-7176_E-mail: kjusoff@yahoo.com
}

This research is partly funded by the Malaysian Government through University Kebangsaan Malaysia under the Science Fund 04-01-02-SF191. We are grateful to Jabatan Ukur dan Pemetaan Malaysia (JUPEM) for providing the GPS data (Sponsoring information)

\begin{abstract}
The free electron distributed in the atmospheric region known as the ionosphere produces a frequency dependent effect on the Global Positioning System (GPS) signals, a delay in the pseudorange and advance in the carrier phase. The ionospheric influence is one of the main problems in the real-time ambiguity resolution for the carrier phase GPS data in radio navigation. Real Time Kinematics (RTK) and Malaysian Active Station (MASS) data from JUPEM (Jabatan Ukur dan Pemetaan Malaysia) were used in this analysis. In this study, the effects of initial phase ambiguity at GPS and modeling of ionosphere on base components were researched. To overcome this problem, a correction ionospheric model was used. This correction model could be implemented in single frequency measurements with similar accuracy, which can be obtained from dual frequency.
\end{abstract}

Keywords: Ambiguity Resolution, Ionosphere, Baseline

\section{Introduction}

The ionospheric delay resulting from radio signals traveling through ionosphere is the major source of errors for single-frequency users of the navigation positioning systems based on satellite. Differential GPS (DGPS) enhances the positional accuracy of GPS receivers. It is based on error correction at the signals for satellite data from ground at known locations. DGPS offers positional accuracy down to centimetre level. It provides single-point error correction data based upon the errors experienced at a single reference station. This is then applied to the mobile receiver, which may be up to several hundred kilometres away. Resolving the GPS carrier-phase ambiguity has been a continuing challenge for sub-centimetre level high precision GPS positioning. In general, centimetre-level GPS positioning 
accuracy requires precise tracking of the carrier phase that consists of two parts: a directly measured fractional part (with measurement error at millimetre level) and an unknown integer part, also called the integer ambiguity. Existing ambiguity resolution techniques can be divided into several categories (Donghyun and Richard Langley, 2000). One of the categories comprises of the most abundant group of techniques, which are based on the theory of integer least squares (Hatch, 1990; Frei and Beutler, 1990; Chen, Lachapelle, 1995; and Teunissen, 1995). Parameter estimation in theory is carried out in three steps, the float solution, the integer ambiguity estimation, and the fixed solution. Each technique makes use of the variance-covariance matrix obtained from the float solution step and employs different ambiguity search processes at the integer ambiguity estimation step.

The integer ambiguity is the unknown integer number of whole cycles between satellite and receiver. The receiver can determine only the fractional part of the wavelength but not the integer, so the ambiguity resolution is essential for precise range determination (Donghyun and Richard Langley; 2000). The goal of ambiguity resolution is to resolve phase ambiguities, i.e. to obtain the correct integer numbers (ambiguity fixing), which is possible at the Double Different (DD) level due to the elimination of instrumental biases etc. So a good ionospheric model is essential in order to get unambiguous results or reduced time to resolve for the ambiguities. Resolution strategies can be divided into those for short baselines and long baselines. The ambiguity resolution, which relies on statistical hypotheses and is highly affected by biases e.g. the ionospheric delay, is difficult to process. After the ambiguities are resolved, the variance ratio is larger and the reference variances are smaller (Abdullah et. al, 2008). This resolution problem is a field of research in itself and a good ionospheric model is essential in some circumstances for good ambiguity resolution.

Generally, most observations for accurate positioning in the network use dual frequency receivers and these are considered expensive to implement in the developing countries. However precise measurements are required in poor countries for purposes such as monitoring land slipping. Not only poor countries could not afford to develop an observation network but even buying one reference dual frequency receiver itself might be difficult. Only single frequency receivers might be affordable to make the relative measurements (Cannon et.al, 1993). So, a correction model that is applicable with single frequency receiver is practical in such situation. In all cases, an accurate ionospheric correction model is essential for most accurate positioning since L1 observations are sensitive to ionospheric error and working on this correction error should be done to support third world countries. An algorithm has been developed in this research that only requires L1 carrier phase measurement (Abdullah, 2004). Some methods have been used to determine the systematic effect due to ionospheric refraction in the L1 carrier of single frequency GPS receiver. While data from dual frequency receivers can account for the ionospheric delay directly by the appropriate linear combination of measurements made on both frequencies, data from single frequency receivers cannot be corrected in this way. The ionospheric model can be used to generate 'correction terms', which can then be applied to the single-frequency observations to account for these effects. The data quality was initially checked using TEQC i.e. the multipath noise (Estey and Meertens, 1999). It is hard to determine the phase multipath that could be only millimetre, where the multipath effect on the phase is two orders of magnitude less than that on the code (Seeber, 2003) where the results obtained are contaminated with multipath. According to Leick (2004), the multipath effect for short baseline was likely cancelled in the single difference observables used in this work. The influence of the correction was examined from the quality check resulting from the data processing and its effects on carrier phase ambiguity resolution errors. The model was evaluated by firstly applying the correction to the PRN 19 measurements, then applying it to the PRN 03, PRN 16, PRN 19 and PRN 1 measurements together.

The integer ambiguity is the unknown integer number of whole cycles between satellite and receiver. The receiver can determine only the fractional part of the wavelength but not the integer, so the ambiguity resolution is essential for precise range determination. The goal of ambiguity resolution is to resolve phase ambiguities, i.e. to obtain the correct integer numbers (ambiguity fixing), which is possible at the Double Different (DD) level due to the elimination of instrumental biases etc. So a good ionospheric model is essential in order to get unambiguous results or reduced time to resolve for the ambiguities. In this paper, the ionospheric correction model proposed for single frequency and the data processing strategy are described and the results obtained in an equatorial region are presented.

\section{Materials and method}

\subsection{GPS data Rinex format}

The developed model was evaluated using real GPS data were obtained from Jabatan Ukur dan Pemetaan Malaysia (JUPEM). The observation data was taken from Universiti Teknologi Malaysia, Johor (1'33' 56.934"N,

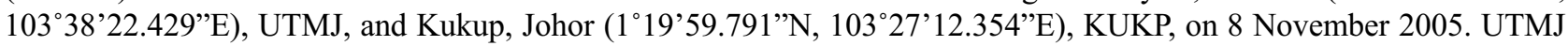
was assumed as a reference station and KUKP was a mobile station giving a baseline of about $33 \mathrm{~km}$.

\subsection{Model application}

The differential ionospheric correction model was applied to GPS carrier phase observables and the results were evaluated. The corrected observation files in RINEX format (Gurtner, 2001) were then used in the processing. GPSurvey software (Trimbel, 1994) was used to process both corrected and uncorrected measurements. In the GPS 
processing software, the quality indicators to evaluate the processing result after the ambiguity is resolved are variance ratio and references variance. They are generated from the statistical results of the observation used. A shorter time was chosen to see how the correction influences the ambiguity resolution. The ambiguity, however, can be resolved for longer periods without applying any ionospheric correction. Both the ratio and the variance distribution can be controlled by the specific level of confidence, which was set to be at $95 \%$. Applying the correction model into the phase measurements should gives better results and this can be evaluated using standard errors of position, ambiguity resolution which is the variance ratio must be larger and the reference variance is smaller.

\section{Results and discussion}

\subsection{Evaluation with PRN 19 measurement corrected}

In this study, only reference station with L1 frequency was used. Within the one hour period from 2:00:00 to 2:59:45, the observed satellites PRNs are 1, 03, 16 and 19 from both stations. The model was evaluated by firstly applying the correction to the PRN 19 measurements, then applying it to the PRN 03, PRN 16, PRN 19 and PRN 1 measurements together.

The ionospheric differential correction was applied to the satellite, namely PRN 19 because this satellite was seen at low elevation angle at the earlier epochs. Firstly, the correction was applied to satellite PRN 19 which had elevation angle of about 28 to 54 degrees.

\subsubsection{Ambiguity not resolved - float solution}

When the processing cannot resolve the ambiguity, it produces a float solution non-integer ambiguity estimate. Figure 1 (a-c) shows that the ambiguity errors of each satellite were plotted. The histogram shows comparison of the errors with uncorrected (without ionospheric correction) and with corrected (with ionospheric correction) measurements at every 10 minutes of processing. The errors are high from the first and decrease to next epochs. (Note1). When the correction was applied to satellite PRN 19, the error decreased a little most of the time but especially for the first 10 minutes of the processing. Figure 2 shows the difference between the errors without correction. When correction was applied in the measurements with respect to the histogram in Fig. 2, it gives an average improvement for PRN 1, followed by PRN 16 and 19. (Note 2). By contrast the standard deviations of baseline components with uncorrected and corrected measurements with float solution are shown in Figure 3. The standard errors of North, East and Up show similar pattern as the ambiguity errors. They reduced over time from about $0.065 \mathrm{~m}$ to $0.015 \mathrm{~m}, 0.6 \mathrm{~m}$ to $0.1 \mathrm{~m}$ and $0.065 \mathrm{~m}$ to $0.2 \mathrm{~m}$ for the North, East and Up respectively. (Note 3, 4). Figure 4 shows that the differences between uncorrected and corrected measurements errors. The East component error reduces to a maximum of about $0.01 \mathrm{~m}$ for the corrected measurements while the other components show smaller reductions. The average reduction with the corrected measurements is about $1 \%$, similar to the difference in the ambiguity errors above.

\subsubsection{Ambiguity resolved- fixed solution}

When the processing can resolve the ambiguity to a correct integer number, it results in a fixed solution. With these 4 satellites, the ambiguities were resolved with the occupation time of 02:54:30. Then by applying the correction model to PRN 19, ambiguities were resolved at 02:53:15 which is about 75 seconds earlier. (Note 5). The improvement can be seen by comparing the variance ratio and reference variance of the processing after the ambiguities were resolved. These are shown in Fig. 5 and Fig. 6 respectively. The ratio in Fig. 5 increases while the reference variance decreases indicating the improvement in the processed results. (Note 6). The difference between the reference variance with no correction and with the correction applied is shown in the Fig. 7. This improvement is quite remarkable by correcting only one satellite in the processing. The difference improves in both the variance ratio and reference variance compared to the uncorrected measurements. (Note 7).

Again, the standard deviations of local position of North, East and Up were compared with the corrected measurements as shown in Fig. 8 (a), (b), and (c). (Note 8) to see how this improvement influences the position. This figure shows a sharp reduction in error compared to that at the time before the ambiguity is resolved as shown in Fig. 2. It can be seen that the standard deviation of the baseline components are changing from the float to fixed solution.

\subsection{Evaluation with PRN 03, PRN 13, PRN 19 And PRN 23 corrected}

Ionospheric corrections were applied to PRN 19, 03, 16 and PRN 01 as well PRN19 for paths with elevation angles of about 28 to 54 degrees, 56 to 76 degrees, 51 to 28 degrees and 57 to 56 degrees respectively. The ambiguity and baseline component errors of the float solution only show improvement with the correction. The duration taken to resolve the ambiguity is reduced to 02:52:30, which is 120 seconds faster.

\subsubsection{Ambiguity not resolved- float solution}

The ambiguity errors of each satellite were plotted in Fig. 9 (a), (b) and (c) in units of cycles. When the processing cannot resolve the ambiguity, it produces a float solution non-integer ambiguity estimate. The histogram shows comparison of the errors with uncorrected and with corrected measurements at every 10 minutes of processing (only 
positive error was plotted in this figure). The errors are high for the first 10 minutes and decrease for the next epochs. During the period from 10 to 50 minutes, the integer part of the decimal ambiguities is not constant. (Note 9,10$)$. When the correction was applied to satellite PRN 19, 3, 16 and PRN 1, the error decreased a little most of the time but especially for the first 20 minutes of the processing. Fig. 10 shows the difference between the errors with no correction and when correction was applied in the measurements with respect to the histogram in Fig. 9. It gives an average improvement for PRN 1, followed by PRN 16 and 19. By contrast the standard deviations of baseline components with uncorrected and corrected measurements with float solution are shown in Fig. 11 (a), (b) and (c) in units of meters. The standard errors of North, East and Up show similar pattern as the ambiguity errors. (Note 11). The differences between uncorrected and corrected measurement errors are shown in Fig. 12. The East component error reduces to a maximum for the corrected measurements while the other components show smaller reductions. The average reduction with the corrected measurements is different in the ambiguity errors above but it still gives an average improvement. (Note 12)

\subsubsection{Ambiguity resolved- fixed solution}

The ratio shows an improvement on average. Even though the ratio increases gradually with the correction, the reference variance does not correspondingly always decrease but increases a little by about 0.2 at 02:57:00 compared to that at 02:55:00. This may be due to other unmodelled errors distorting the solution such as multipath. (Note 13, 14). Both the variance ratio and the reference variance are illustrated in Fig. 15 and show significant improvement with corrected measurements compared to Fig. 13 and Fig.14. The difference between the reference variance with no correction and with correction is shown in Fig. 15 with an increasing trend. (Note 15). Again the standard deviations of local position of North, East and Up were compared with corrected measurements as shown in Figure 16. (Note 16) to see how this improvement influences the position. Figure 16 shows a sharp reduction in error compared to that at the time before the ambiguity is resolved as shown in Fig.9. It can be seen that the standard deviation of the baseline components is changing from the float to fixed solution. Table 1 summarises the ambiguity resolution success rate without and with the correction applied. When the processing cannot resolve the ambiguity, it produces a float solution noninteger ambiguity estimate. Whereas, when the processing can resolve the ambiguity to a correct integer number, it results in a fixed solution. With these 4 satellites, (uncorrected data) the ambiguities were resolved with the occupation time of 02:54:30. By applying the correction model to PRN 19, ambiguities were resolved at 02:53:15, which is 1 minute $15 \mathrm{sec}$ earlier corresponding to uncorrected data and when the correction model was applied to PRN 03, 13, 19 \& 23, ambiguities were resolved at 02:52:30 which is 1 minute $15 \mathrm{sec}$ earlier corresponding to corrected data with PRN 19 only and two minute earlier compared to four satellites (uncorrected data). (Note 17)

The effectiveness of this new technique has been determined by implementing it into real GPS data for short and long baselines. In order to obtain the absolute value of the differential ionospheric delay from the measurements, integer ambiguities have to be resolved. Processing software, have been used to accomplish this task, therefore the model can be validated. The modeled ionospheric delays show good correlation with the computed absolute delay. However, one should note that, in the real world environment, the mobile receivers can be anywhere, such as between the high buildings where multipath is high, which will contaminate the ionospheric induced delay.

Finally, by applying the corrected model, the obtained results compared to uncorrected measurements have indicated that the ambiguity success rate is faster even when only correcting one satellite seen at low elevation angles. This success rate increases by correcting other satellite paths. After the ambiguities are resolved, the variance ratio is larger and the reference variances are smaller. This implies good processing results.

\section{Conclusions}

In this study, the developed differential ionosphere correction model has been evaluated using real GPS measurement. Then the specific topics were considered: float and fixed solution of ambiguity resolution and then effects on the estimated baseline components. This was done by processing the uncorrected and corrected measurements for the given satellites. It was shown that by applying the correction model, the success of ambiguity resolution was achieved earlier when almost all measurements were corrected. For positioning, the standard deviation with respect to the local geodetic component of North, East and Up are significantly reduced.

Implementing this work further would extend the ionospheric correction model to longer baselines for relative measurements at any location with any ionospheric conditions by using only single frequency receivers together with one reference dual frequency receiver. The outcome gives a different approach that could be considered also for current or future GNSS augmentation systems to overcome the ionospheric error.

\section{References}

Abdullah, M., H.J. Strangeways \& D.M.A.Walsh. (2008). Improving ambiguity resolution rate with an accurate ionospheric differential correction. Journal of Navigation-Cambridge Univ. Press, 62 (1), - accepted for publication.

Abdullah, M. (2004). Modelling and Determination of Ionospheric Effect on Relative on GPS Measurements. The University of Leeds School of Electronic and Electrical Engineering. 
Cannon M.E., Lachapelle, G., Lu, G. (1993). Kinematic Ambiguity Resolution with a High Precision C/A Code Receiver", Department of Geomatics Engineering, University of Calgary Calgary, Alberta. Journal of Surveying Engineering, Amer. Soc. Civil Eng, Vol. 119, No. 4 (November).

Chen D, Lachapelle G. (1995). A Comparison of the FASF and Least-Squares Search Algorithms for On-the-Fly Ambiguity Resolution. Navig: J Inst Navig.

Donghyun K., and Richard B. Langley. (2000). GPS Ambiguity Resolution and Validation: Methodologies, Trends and Issues. International Symposium on GPS/GNSS, Seoul.

Estey, L. H., Meertens, C. M. (1999). TEQC: the multi-purpose toolkit for GPS/GLONASS data, GPS Solutions, 3(1), $42-49$.

Frei E, Beutler G. (1990). Rapid Static Positioning Based on the Fast Ambiguity Resolution Approach FARA: Theory and First Result, Manuscr Geod.

Gurtner, W. (2001). RINEX: The Receiver Independent Exchange Format Version 2.10. [Online] Available: http://www.ngs.noaa.gov/CORS/Rinex2.html, accessed on 20 July 2008.

Hatch H. (1990). Instantaneous Ambiguity Resolution, Proceeding of KIS90, Banf.

Leick, A. (2004). GPS Satellite Surveying. Wiley, New Jersey.

Seeber,G.. (2003). Satellite Geodesy. Walter de Gruyter, Berlin.

Teunissen P.J.G. (1995). A New Method for Fast Carrier Phase Ambiguity Estimation. Proceeding of IEEE Position, Location and Navigation Symposium, Las Vegas.

Trimble. (1994). WAVE software user's guide. Sunnyvale,CA,USA: Trimble Navigation Limited

Table 1. Ambiguity resolution success rate

\begin{tabular}{|l|l|l|}
\hline \multirow{2}{*}{$\begin{array}{l}\text { Without } \\
\text { Correction }\end{array}$} & \multicolumn{2}{|l|}{ With Correction } \\
\cline { 2 - 3 } & PRN 19 & $\begin{array}{l}\text { PRN 03, 13, 19 \& } \\
23\end{array}$ \\
\hline $02: 54: 30$ & $02: 53: 15$ & $02: 52: 30$ \\
\hline
\end{tabular}

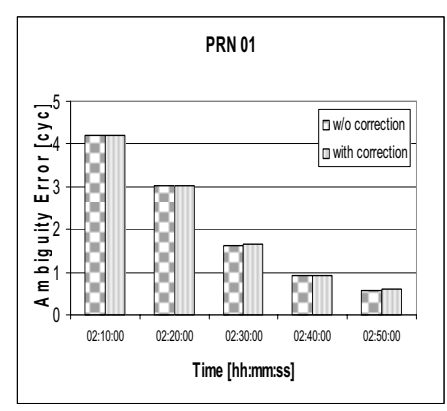

(a)

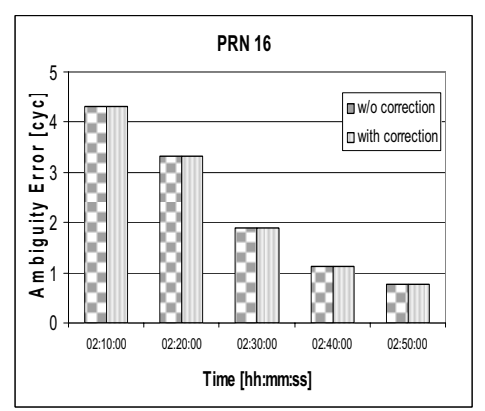

(b)

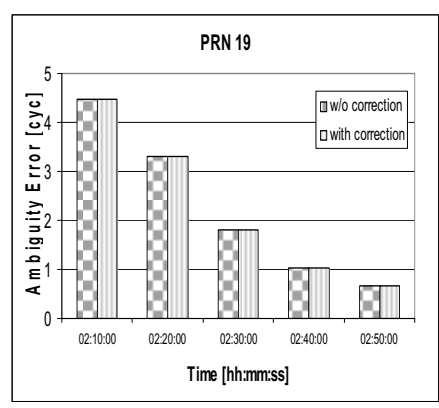

(c)

Figure 1. Ambiguity error of float solution for UTMJ312 and KUKP312

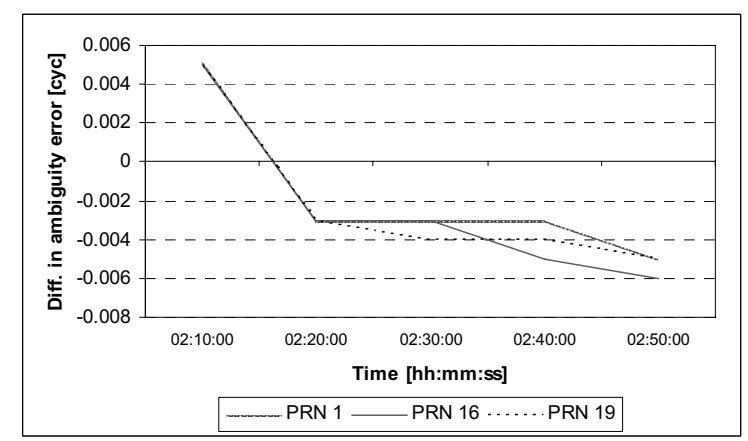

Figure 2. The difference in the ambiguity errors of float solution 


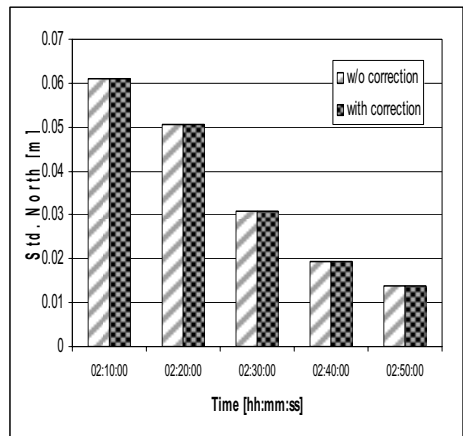

(a)

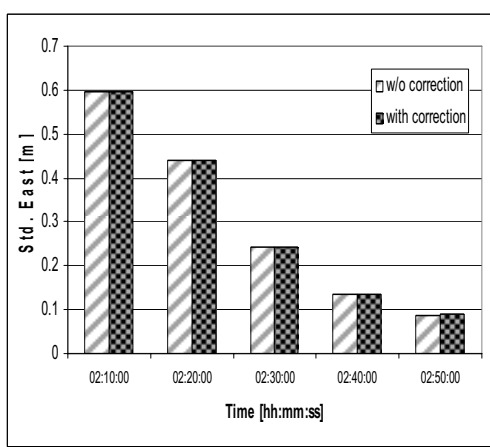

(b)

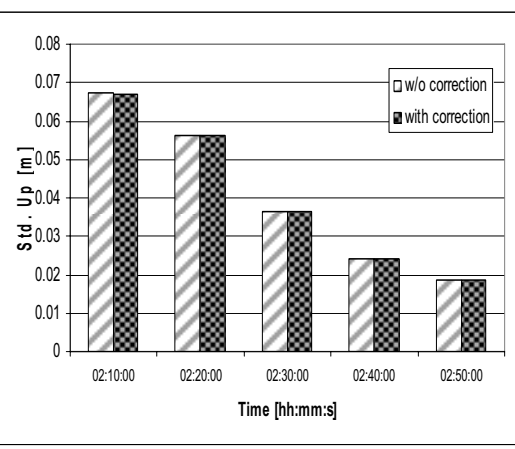

(c)

Figure 3. Standard errors of baseline components (float solution) for UTMJ312 and KUKP312

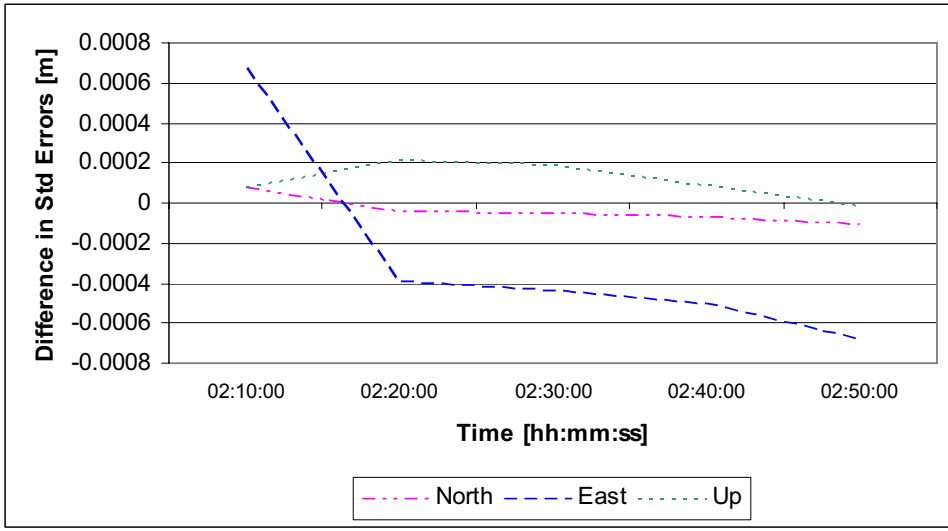

Figure 4. The difference in standard error of float solutions

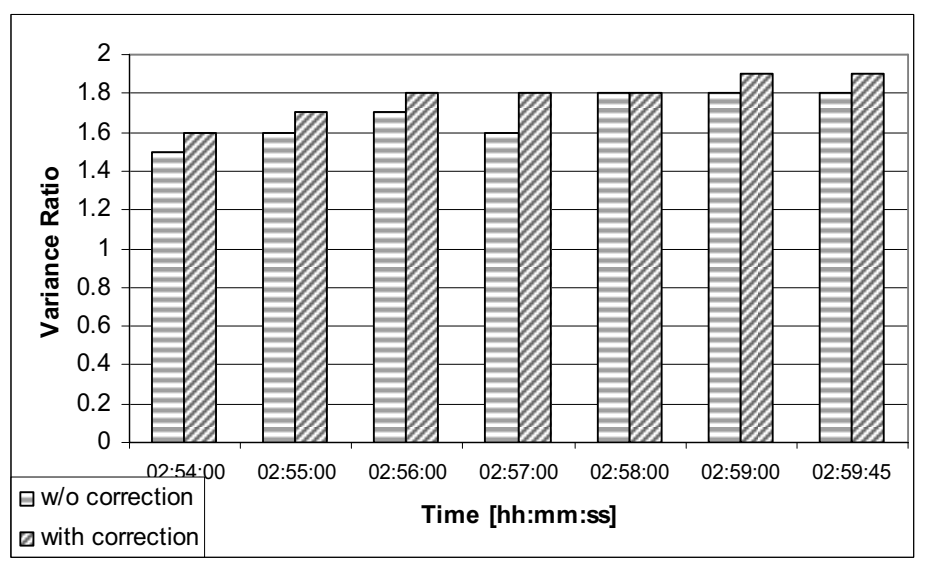

Figure 5. Comparison of Variance Ratio for UTMJ312 and KUKP312 


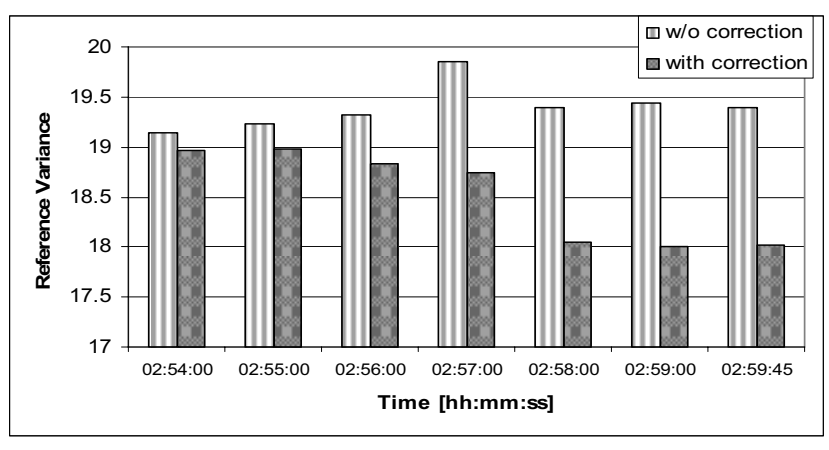

Figure 6. Comparison of Reference Variance for UTMJ312 and KUKP312

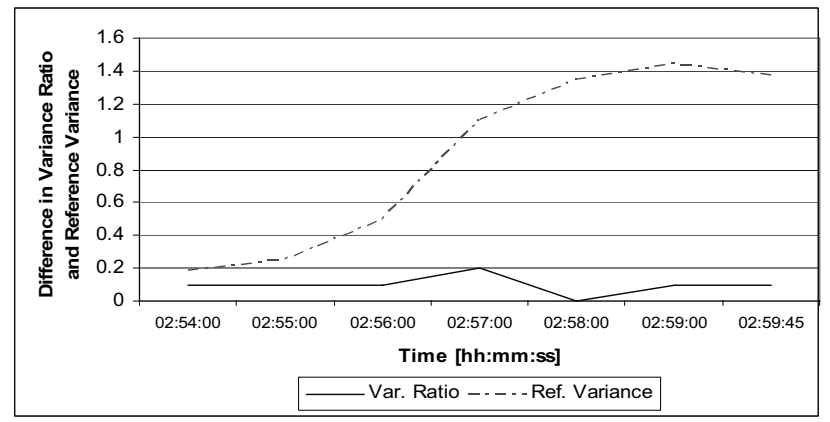

Figure 7. Difference in the reference variance and variance ratio for UTMJ312 and KUKP312

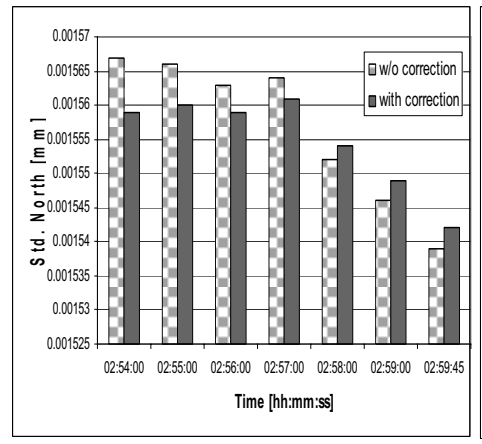

(a)

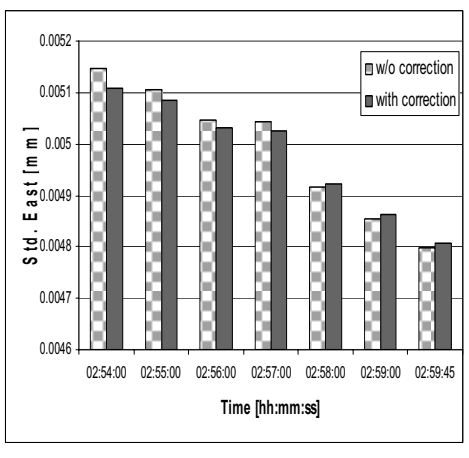

(b)

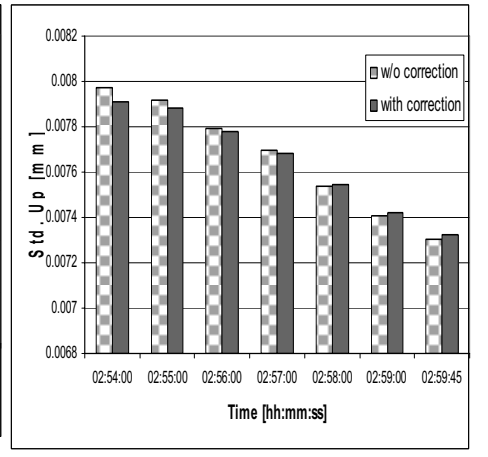

(c)

Figure 8. Standard errors of baseline components (fixed solution) for UTMJ312 and KUKP312

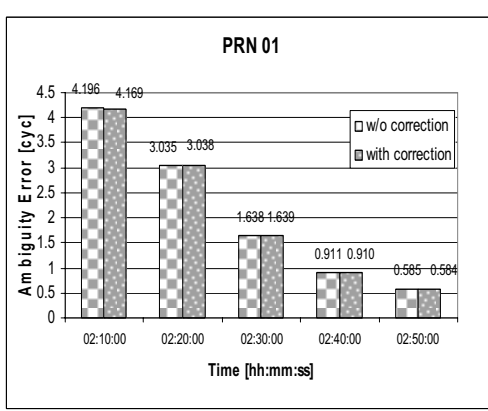

(a)

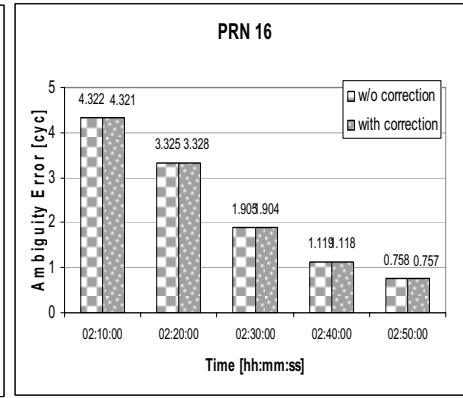

(b)

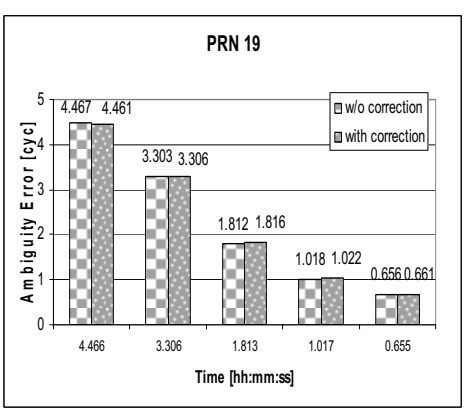

(c)

Figure 9. Ambiguity error of float solution for UTMJ312 and KUKP312 


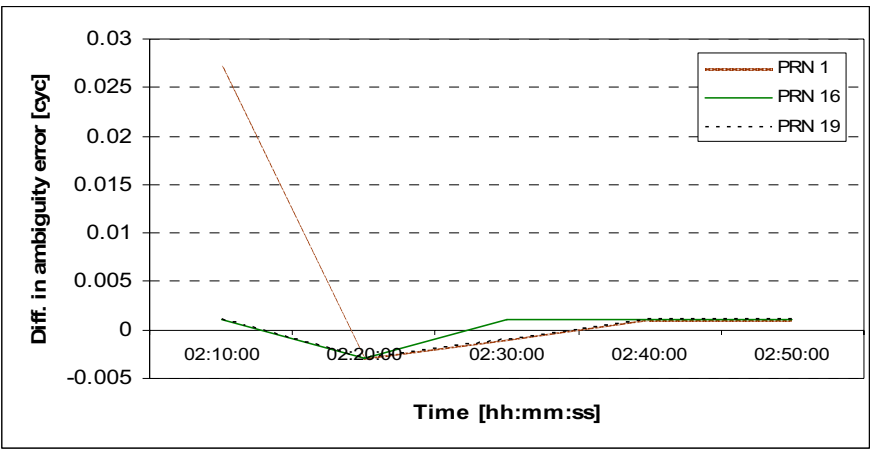

Figure 10. The difference in the ambiguity error of float solution for UTMJ312 and KUKP312

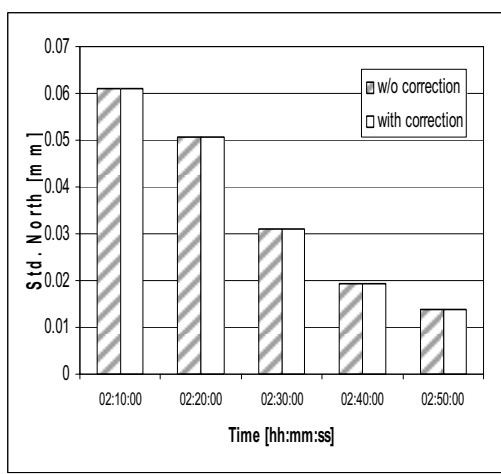

(a)

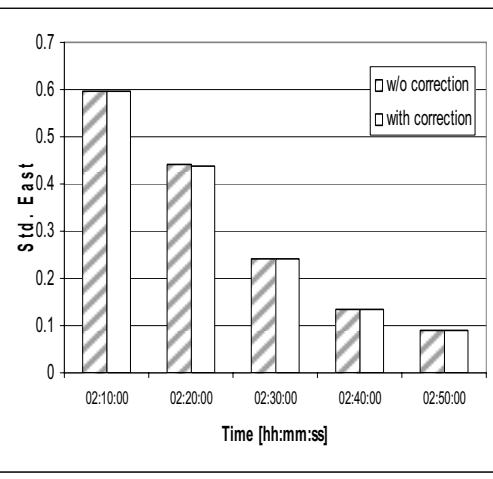

(b)

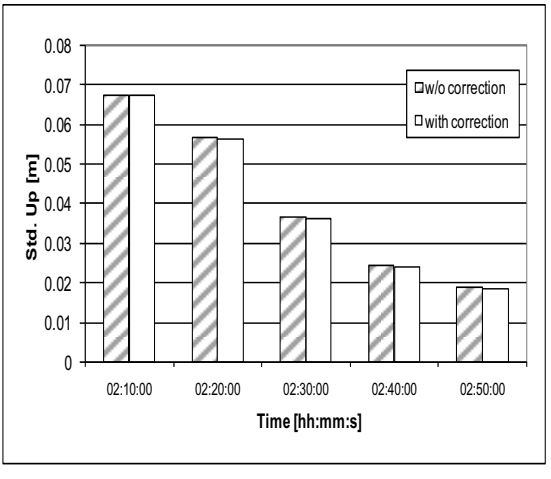

(c)

Figure 11. Standard errors of baseline components (float solution) for UTMJ312 and KUKP312

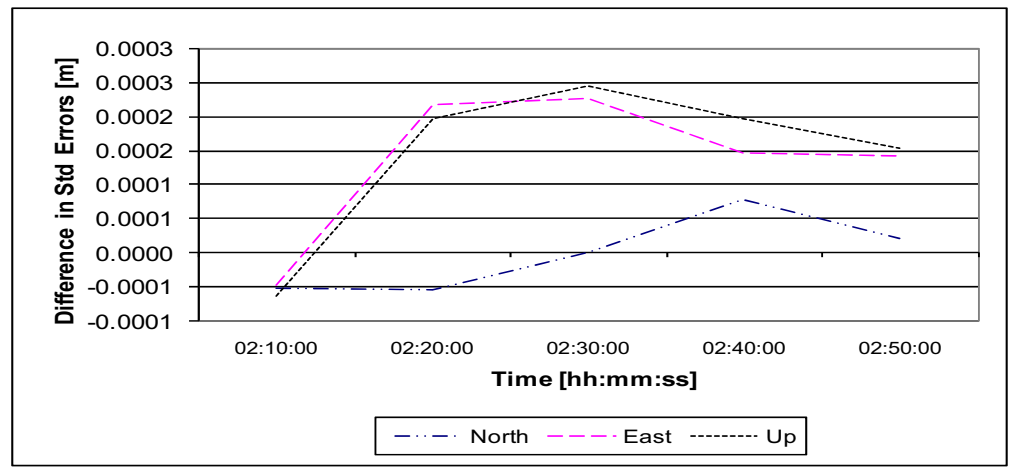

Figure 12. Difference in the standard errors (float solution) for UTMJ312 and KUKP312

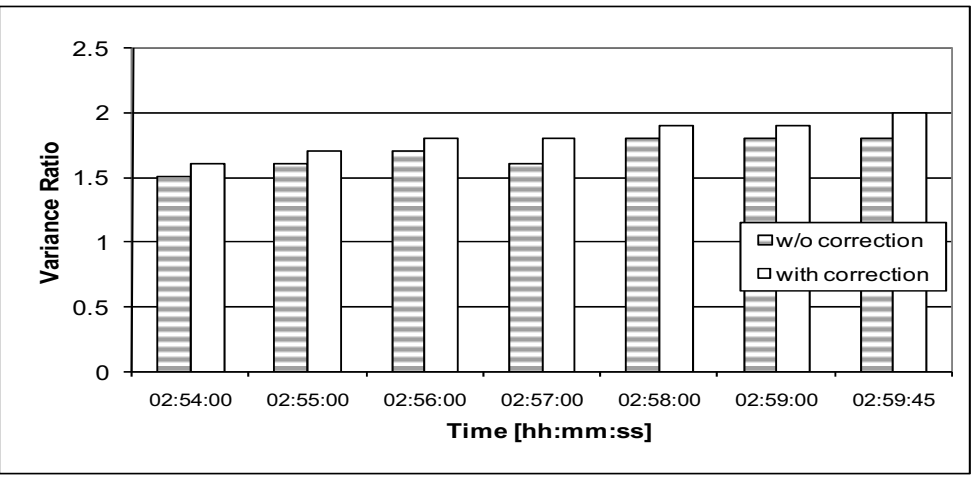

Figure 13. Comparison of Variance ratio for UTMJ312 and KUKP312 


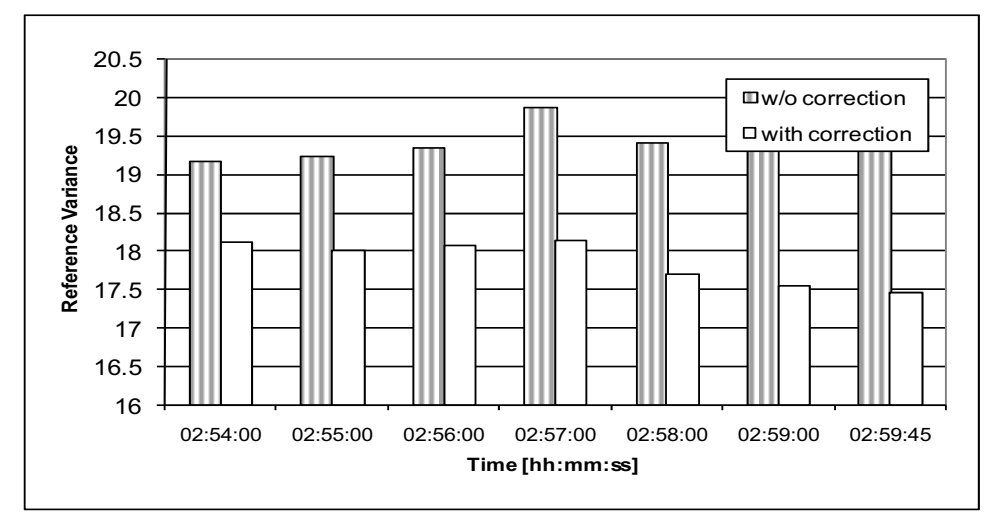

Figure 14. Comparison of Reference Variance for UTMJ312 and KUKP312

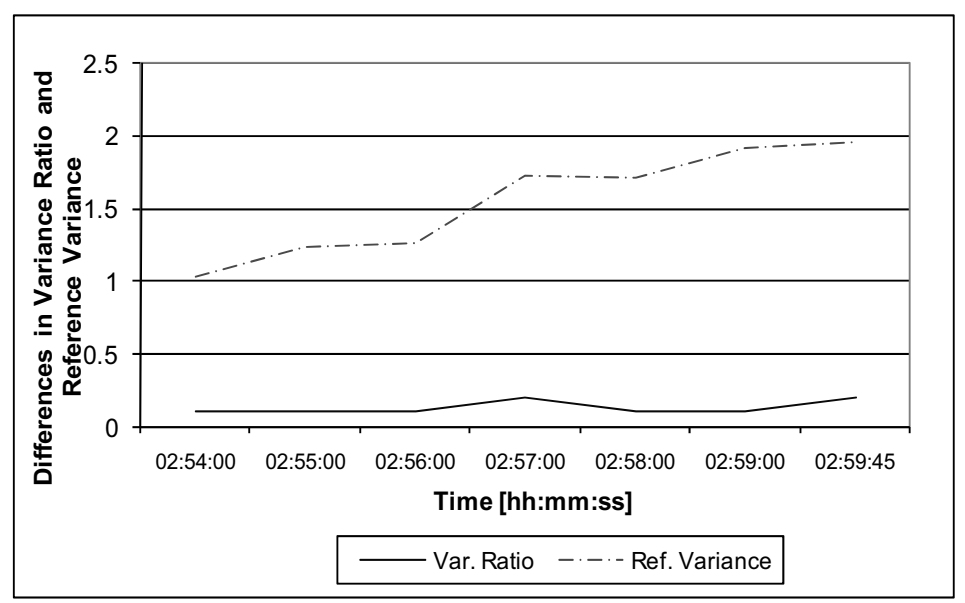

Figure 15. Differences in Variance ratio and Reference Variance for UTMJ312 and KUKP312

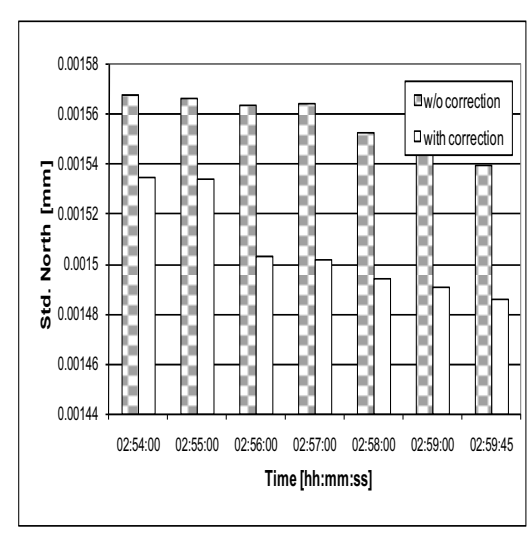

(a)

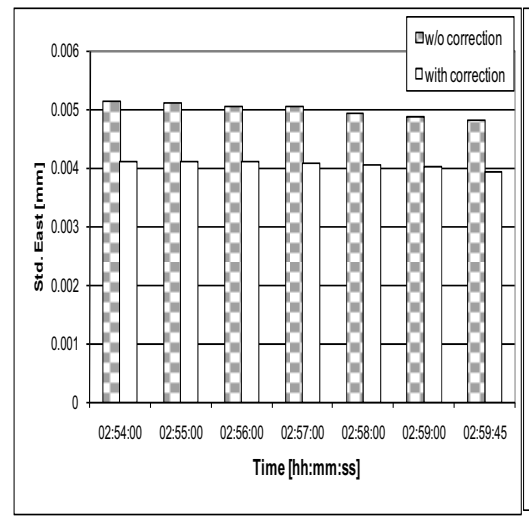

(b)

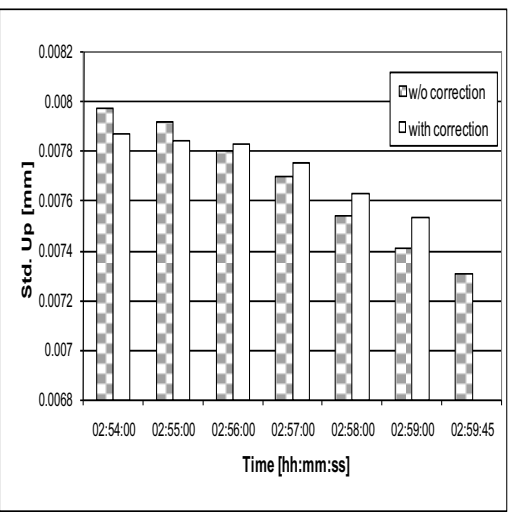

(c)

Figure 16. Standard errors of baseline components (fixed solution) for UTMJ312 and KUKP312 